T. E. CECIL

KODAI MATH. J.

13 (1990), 143-153

\title{
ON THE LIE CURVATURE OF DUPIN HYPERSURFACES
}

\author{
By Thomas E. Cecil
}

\section{Introduction.}

A hypersurface $M$ in a standard sphere $S^{n}$ is said to be Dupin if each of its principal curvatures is constant along its corresponding curvature surfaces. If the number of distinct principal curvatures is constant, then $M$ is called a proper Dupin hypersurface. There is a close relationship between the class of compact proper Dupin hypersurfaces and the class of isoparametric hypersurfaces. Münzner [11] showed that the number $g$ of distinct principal curvatures of an isoparametric hypersurface must be $1,2,3,4$ or 6 . Thorbergsson [15] then showed that the same restriction holds for a compact proper Dupin hypersurface embedded in $S^{n}$ by reducing that case to a situation where Münzner's argument can be applied. This also implied that the rank of the $Z_{2}$-cohomology ring in both cases must be $2 g$. Later Grove and Halperin [6] found more topological similarities between these two classes of hypersurfaces. All of this led to the conjecture [5, p. 184] that every compact proper Dupin hypersurface in $S^{n}$ is equivalent by a Lie sphere transformation to an isoparametric hypersurface.

The conjecture was known to be true in the cases $g=1$ (umbilic hypersurfaces), $g=2[4]$ and $g=3[7]$. Recently, however, counterexamples to the conjecture for $g=4$ have been discovered by Miyaoka and Ozawa [10] and by Pinkall and Thorbergsson [14]. Miyaoka and Ozawa also produced counterexamples in the case $g=6$. In all cases, the proof that the counterexamples are not Lie equivalent to an isoparametric hypersurface uses the so-called Lie curvature $\Psi$ introduced by Miyaoka [8]. For a proper Dupin hypersurface $M$ with four principal curvatures, $\Psi$ is the cross-ratio of these principal curvatures. Viewed in the context of Lie sphere geometry, $\Psi$ is the cross-ratio of the four points on a projective line corresponding to the four curvature spheres of $M$. Hence, $\Psi$ is a natural Lie invariant. From Münzner's work, it is easy to compute that $\Psi$ has a constant value $1 / 2$ on a Dupin hypersurface which is Lie equivalent to an isoparametric hypersurface. In projective geometric terms, this means that the four curvature spheres at each point of $M$ form a harmonic set. For the counterexamples to the conjecture above, $\Psi$ does not have the constant value $1 / 2$.

Research supported by NSF Grant No. DMS-8907366.

Received September 1, 1989 
The converse problem involves the strength of the assumption that $\Psi=1 / 2$ on $M$. Miyaoka [8] proved that the assumption that $\Psi$ is constant on a compact proper Dupin hypersurface together with an additional assumption regarding intersections of leaves of the principal foliations implies that $M$ is Lie equivalent to an isoparametric hypersurface. In this note, we show that some global hypotheses are necessary to reach Miyaoka's conclusion by exhibiting a non-compact proper Dupin hypersurface in $S^{n}$ on which $\Psi=1 / 2$ which is not Lie equivalent to an open subset of an isoparametric hypersurface in $S^{n}$. We also produce examples on which $\Psi$ has a constant value $c, 0<c<1$. These are the only values possible for $\Psi$ under Miyaoka's definition. These examples are all obtained as follows. Begin with an isoparametric hypersurface $V$ with three principal curvatures in a sphere $S^{n-m}$. Embed $S^{n-m}$ as a totally geodesic submanifold in $S^{n}$, and take a tube of constant radius over $V$ in $S^{n}$. The examples are all open subsets of such tubes.

\section{Dupin and isoparametric hypersurfaces in Lie sphere geometry.}

In this section, we begin by briefly outlining the framework for the study of Dupin hypersurfaces in Lie sphere geometry. The notation is taken from [2] which provides more details. At the end of this section, we prove an elementary result, Theorem 1 , which is essentially a Lie geometric formulation of the concept of an isoparametric hypersurface.

Let $\boldsymbol{R}_{2}^{n+3}$ be a real vector space of dimension $n+3$ endowed with a metric of signature $(n+1,2)$,

$$
\langle x, y\rangle=-x_{1} y_{1}+x_{2} y_{2}+\cdots+x_{n+2} y_{n+2}-x_{n+3} y_{n+3} .
$$

Let $e_{1}, \cdots, e_{n+3}$ denote the standard orthonormal basis for this metric with $e_{1}$ and $e_{n+3}$ timelike. Let $P^{n+2}$ be the real projective space of lines through the origin in $\boldsymbol{R}_{2}^{n+3}$, and $Q^{n+1}$ the so-called Lie quadric hypersurface in $P^{n+2}$ given by the equation $\langle x, x\rangle=0$. Finally, let $S^{n}$ be the unit sphere in the Euclidean space $\boldsymbol{R}^{n+1}$ spanned by the vectors $e_{2}, \cdots, e_{n+2}$.

Lie sphere geometry gives a bijective correspondence between the points of $Q^{n+1}$ and the set of all oriented hyperspheres and point spheres in $S^{n}$ (see [2]). The hypersphere with center $p \in S^{n}$ and signed radius $\rho$ corresponds to the point $[(\cos \rho, p, \sin \rho)]$ in $Q^{n+1}$, where the square brackets denote the point in $P^{n+2}$ given by the homogeneous coordinates within the round brackets. The point spheres in $S^{n}$ correspond to those points with $\rho=0$. From this relationship, one easily computes that a point $[x] \in Q^{n+1}$ corresponds to an oriented hypersphere of signed radius $\rho$ if and only if

$$
\left\langle x, \sin \rho e_{1}-\cos \rho e_{n+3}\right\rangle=0 .
$$

The Lie quadric contains projective lines but no linear subspaces of $P^{n+2}$ of higher dimension. The line $[x, y]$ determined by two points $[x]$ and $[y]$ of 
$Q^{n+1}$ lies on $Q^{n+1}$ if and only if $\langle x, y\rangle=0$. Geometrically, this means that the two hyperspheres in $S^{n}$ corresponding to the points $[x]$ and $[y]$ are in oriented contact. The points on a line on the quadric correspond to the pencil of oriented spheres in oriented contact at a point $(p, \xi)$ in the unit tangent bundle to $S^{n}$. We call $(p, \xi)$ a contact element.

A Lie sphere transformation is a projective transformation of $P^{n+2}$ which takes $Q^{n+1}$ to itself. Since it takes lines on $Q^{n+1}$ to lines on $Q^{n+1}$, a Lie sphere transformation preserves oriented contact of spheres. The group of Lie sphere transformations is isomorphic to $O(n+1,2) /\{ \pm I\}$, where $O(n+1,2)$ is the orthogonal group for the metric (1.1).

The manifold $\wedge^{2 n-1}$ of lines on $Q^{n+1}$ has a contact structure, i. e., a globally defined 1-form $\omega$ such that $\omega \wedge d \omega^{n-1}$ never vanishes on $\wedge^{2 n-1}$. The condition $\omega=0$ defines a codimension one distribution $D$ on $\wedge^{2 n-1}$ which has integral submanifolds of dimension $n-1$, but none of higher dimension. An immersion $\lambda: M^{n-1} \rightarrow \wedge^{2 n-1}$ such that $\lambda^{*} \omega=0$ is called a Legendre submanifold [2] or Lie geometric hypersurface [12]. If $\alpha$ is a Lie sphere transformation, then $\alpha \lambda$ : $M^{n-1} \rightarrow \wedge^{2 n-1}$ is also a Legendre submanifold. It is said to be Lie equvvalent to $\lambda$. Pinkall [12] shows that giving a Legendre submanifold $\lambda$ is equivalent to giving two functions $k_{1}, k_{2}$ from $M$ to $\boldsymbol{R}_{2}^{n+3}$ such that:

(L1) For all $x \in M$, the vectors $k_{1}(x)$ and $k_{2}(x)$ are linearly independent

and

$$
\left\langle k_{\imath}, k_{\jmath}\right\rangle=0, i, j=1,2 .
$$

(L2) There is no $x \in M, X \in T_{x} M$ such that $d k_{1}(X)$ and $d k_{2}(X)$ are both in Span $\left\{k_{1}(x), k_{2}(x)\right\}$.

$$
\left\langle d k_{1}, k_{2}\right\rangle=0 .
$$

The Legendre submanifold is then defined by $\lambda(x)=\left[k_{1}(x), k_{2}(x)\right]$. Conditions (L1)-(L3) are preserved if one reparametrizes by taking $\tilde{k}_{1}=\alpha k_{1}+\beta k_{2}$ and $\tilde{k}_{2}=$ $\gamma k_{1}+\delta k_{2}$, where $\alpha, \beta, \gamma, \delta$ are smooth real-valued functions on $M$ with $\alpha \delta-\beta \gamma$ never zero.

An immersion $f: M^{n-1} \rightarrow S^{n}$ with field of unit normals $\xi: M^{n-1} \rightarrow S^{n}$ naturally induces a Legendre submanifold defined by the two functions

$$
k_{1}=(1, f, 0), \quad k_{2}=(0, \xi, 1) .
$$

For each $x \in M,\left[k_{1}(x)\right]$ is the point sphere on the line $\lambda(x)$, and $\left[k_{2}(x)\right]$ is the great sphere on $\lambda(x)$. An immersed submanifold $\varphi: V \rightarrow S^{n}$ of codimension greater than one also induces a Legendre submanifold whose domain is the bundle $B^{n-1}$ of unit normal vectors to $\varphi(V)$. For a unit normal $\xi$ to $\varphi(V)$ at a point $\varphi(v)$, we define $\lambda(v, \xi)$ to be the line on $Q^{n+1}$ corresponding to the pencil of spheres in oriented contact at the contact element $(\varphi(v), \xi)$. In this case, the point sphere map $k_{1}(v, \xi)=(1, \varphi(v), 0)$ has constant rank equal to the dimension of $V$. For a general Legendre submanifold, the point sphere map is not an immersion, nor does it have constant rank. It can always be written in the form (1.3), 
and the map $f$ is called the spherical projection of the Legendre submanifold.

Suppose that $\lambda: M^{n-1} \rightarrow \wedge^{2 n-1}$ is a Legendre submanifold determined by $k_{1}$ and $k_{2}$ satisfying (L1)-(L3). A sphere $K=\alpha k_{1}+\beta k_{2}$ is called a curvature sphere at $x \in M$ if there is a non-zero vector $X \in T_{x} M$ such that $\alpha d k_{1}(X)+\beta d k_{2}(X)$ is in Span $\left\{k_{1}(x), k_{2}(x)\right\}$. The vector $X$ is called a principal vector corresponding to $K$. If $k_{1}$ and $k_{2}$ are given as in (1.3) by an immersed hypersurface in $S^{n}$, then the curvature spheres at $x$ are precisely those hyperspheres in oriented contact with the hypersurface at $f(x)$ which are centered at the focal points along the normal geodesic to the hypersurface at $f(x)$. The principal vectors are just the usual principal vectors for the shape operator of the immersion. For an arbitrary Legendre submanifold, the principal vectors corresponding to a given curvature sphere form a subspace of $T_{x} M$, and $T_{x} M$ is the direct sum of these principal spaces. Further, if a continuous curvature sphere $K$ has constant multiplicity on $M$, then its principal distribution is a smooth foliation. If the multiplicity of $K$ is greater than one, then $K$ is constant along the leaves of its principal foliation.

A connected submanifold $N$ of $M$ is called a curvature surface if its tangent space is everywhere a principal space, e.g., a leaf of a principal foliation. A Legendre submanifold is called Dupin if along each curvature surface, the corresponding curvature sphere is constant. A Dupin submanifold is said to be proper if the number of distinct curvature spheres is constant. The Legendre submanifold induced from a Dupin hypersurface in $S^{n}$ is Dupin in the sense defined here, but our definition is more general, since it is not necessary for the spherical projection to be an immersion. Both the Dupin and proper Dupin properties are easily seen to be Lie invariant.

We now give an elementary Lie geometric characterization of those proper Dupin submanifolds which are Lie equivalent to the Legendre submanifold induced by an isoparametric hypersurface in $S^{n}$. Compactness is not required for this result, i.e., the theorem characterizes those Dupin submanifolds which are Lie equivalent to open subsets of compact isoparametric hypersurfaces.

Recall that a line in $P^{n+2}$ is called timelike if it contains only timelike points. This means that an orthonormal basis for the 2-plane in $\boldsymbol{R}_{2}^{n+3}$ determined by the projective line consists of two timelike vectors. An example is the line $\left[e_{1}, e_{n+3}\right]$.

THEOREM 1. Let $\lambda: M^{n-1} \rightarrow \wedge^{2 n-1}$ be a proper Dupin submanifold with $g$ distinct curvature spheres $K_{1}, \cdots, K_{g}$ at each point. Then $\lambda$ is Lie equivalent to the Legendre submanifold induced by an isoparametric hypersurface in $S^{n}$ if and only if there exist $g$ points $P_{1}, \cdots, P_{g}$ on a timelike line in $P^{n+2}$ such that

$$
\left\langle K_{\imath}, P_{\imath}\right\rangle=0, \quad 1 \leqq i \leqq g .
$$

Proof. If $\lambda$ is the Legendre submanifold induced by an isoparametric hypersurface, then the spheres in each family $K_{\imath}$ all have the same radius $\rho_{i}$. Thus, by (1.2) we have $\left\langle K_{\imath}, P_{\imath}\right\rangle=0$, where the $P_{\imath}$, 


$$
P_{\imath}=\sin \rho_{i} e_{1}-\cos \rho_{2} e_{n+3}, \quad 1 \leqq i \leqq g,
$$

are $g$ points on the timelike line $\left[e_{1}, e_{n+3}\right]$. Since a Lie transformation preserves curvafure spheres, timelike lines and the polarity relationship, the same is true for any Lie image of $\lambda$.

Conversely, suppose that there exist points $P_{1}, \cdots, P_{g}$ on a timelike line $l$ such that $\left\langle K_{2}, P_{2}\right\rangle=0$. Let $\alpha$ be a Lie transformation which takes $l$ to the line $\left[e_{1}, e_{n+3}\right]$. Then the curvature spheres $\alpha K_{2}$ of the Dupin submanifold $\alpha \lambda$ are respectively orthogonal to the points $Q_{\imath}=\alpha P_{\imath}$ on the line $\left[e_{1}, e_{n+3}\right]$. This means that for each $i$, the spheres $\alpha K_{\imath}$ have constant radius on $M$. That is, $\alpha \lambda$ is induced from an isoparametric hypersurface in $S^{n}$.

Remark 1. In the case where $\lambda$ is Lie equivalent to an isoparametric hypersurface, one can say more about the position of the points $P_{1}, \cdots, P_{g}$ along the line $l$. Münzner [11] showed that radii $\rho_{i}$ of the curvature spheres of an isoparametric hypersurface must be of the form

$$
\rho_{i}=\rho_{1}+(i-1) \pi / g, \quad 1 \leqq i \leqq g,
$$

for some $\rho_{1}$ in $(0, \pi / g)$. Hence, after Lie transformation, the $P_{\imath}$ must have the form (1.4) for $\rho_{\imath}$ as in (1.5).

Remark 2. Theorem 1 could be used to significantly shorten the proof of the classification of Dupin hypersurfaces with $g=3$ in $S^{4}$ given by Chern and the author [3] (see also Pinkall [13]). In that proof, the three curvature spheres are given by maps $Y_{1}, Y_{7}$ and $Y_{1}+Y_{7}$ from $\boldsymbol{M}^{3}$ to the Lie quadric $\boldsymbol{Q}^{5}$. In the treatment of the case $\rho \neq 0[3$, pp. 39-40], we show by equations (5.79)-(5.81) that the three curvature spheres satisfy

$$
\left\langle Y_{1}, W_{1}\right\rangle=0, \quad\left\langle Y_{7}, W_{2}\right\rangle=0, \quad\left\langle Y_{1}+Y_{7}, W_{1}-W_{2}\right\rangle=0,
$$

for three points on the timelike line $\left[W_{1}, W_{2}\right]$. At that point in the proof, Theorem 1 could have been invoked to conclude that $M^{3}$ is Lie equivalent to an open subset of an isoparametric hypersurface. Instead, we gave a longer direct proof that $M^{3}$ is Lie equivalent to an open subset of a tube over a Veronese surface in $S^{4}$, thereby, in effect, reproving Cartan's classification of isoparametric hypersurfaces with $g=3$ in $S^{4}$.

\section{Lie curvature of Dupin submanifolds.}

Let $\lambda: M^{n-1} \rightarrow \wedge^{2 n-1}$ be a proper Dupin submanifold with $g$ distinct curvature spheres $K_{1}, \cdots, K_{g}$ at each point. Let $k_{1}=(1, f, 0)$ and $k_{2}=(0, \xi, 1)$ be the point sphere and great sphere maps, respectively, determined by $\lambda$. At each point $x \in M$, we can represent the points on the line $\lambda(x)$ in the form $\mu k_{1}(x)+$ $k_{2}(x)$, i. e., take $\mu$ as an inhomogeneous coordinate along the line $\lambda(x)$. Of course, $k_{1}(x)$ corresponds to $\mu=\infty$. In particular, we write the curvature 
spheres as

$$
K_{\imath}=\mu_{\imath} k_{1}+k_{2}, \quad 1 \leqq i \leqq g .
$$

Assume now that $g \geqq 4$. Miyaoka [8] pointed out that if $\sigma$ is any permutation of the numbers $1, \cdots, g$, then the cross-ratio

$$
\left(\mu_{\sigma(4)}-\mu_{\sigma(3)}\right)\left(\mu_{\sigma(1)}-\mu_{\sigma(2)}\right) /\left(\mu_{\sigma(4)}-\mu_{\sigma(2)}\right)\left(\mu_{\sigma(1)}-\mu_{\sigma(3)}\right)
$$

of the four curvature spheres $K_{\sigma(1)}, \cdots, K_{\sigma(4)}$ is invariant under Lie sphere transformations. All of these cross-ratios are called Lie curvatures of $\lambda$. In the case $g=4$, we can order the $\mu_{2}$ so that $\mu_{1}<\mu_{2}<\mu_{3}<\mu_{4}$. Then there is one uniquely defined Lie curvature function $\Psi$ given by

$$
\Psi=\left(\mu_{4}-\mu_{3}\right)\left(\mu_{1}-\mu_{2}\right) /\left(\mu_{4}-\mu_{2}\right)\left(\mu_{1}-\mu_{3}\right) .
$$

Suppose that the spherical projection $f$ is an immersion at a point $x \in M$, i. e., $k_{1}(x)$ is not a curvature sphere. For $X \in T_{x} M$ and $\mu \in \boldsymbol{R}$, we have

$$
d\left(\mu k_{1}+k_{2}\right)(X)=(0, \mu d f(X)+d \xi(X), 0) .
$$

The vector on the right is in $\operatorname{Span}\left\{k_{1}(x), k_{2}(x)\right\}$ if and only if $d \xi(X)=-\mu d f(X)$, i. e., $\mu$ is a principal curvature of the immersion $f$ at $x$. Thus, the cross-ratio $\Psi$ is also the cross-ratio of the principal curvatures. If $k_{1}(x)$ is a curvature sphere, then by applying a parallel transformation $P_{t}$ (see [2] and [12]) to $\lambda$, one can obtain a Legendre submanifold $P_{t} \lambda$ whose spherical projection $f_{t}=$ $\cos t f+\sin t \xi$ is an immersion at $x$. Then the cross-ratio (2.2) of four curvature spheres of $\lambda$ at $x$ is equal to the cross-ratio of the corresponding four curvature spheres of $P_{t} \lambda$. This, in turn, is equal to the cross-ratio of the corresponding four principal curvatures of the immersion $f_{t}$ at $x$.

A noteworthy special case is when the point sphere map $k_{1}$ is a curvature sphere of multiplicity $m$ on $M$. Then the spherical projection of $\lambda$ factors though an immersion $\varphi$ of the $(n-1-m)$-dimensional space of leaves $V$ of the corresponding principal foliation. Furthermore, $M$ is diffeomorphic to the bundle of unit normals to the immersed submanifold $\varphi(V)$ of codimension $m+1$ in $S^{n}$, and $\lambda$ can be considered to be the Legendre submanifold induced by the submanifold $\varphi(V)$, as discussed in Section 1.

The paper [14] of Pinkall and Thorbergsson suggests the following treatment of the case of a Legendre submanifold $\lambda: B^{n-1} \rightarrow \wedge^{2 n-1}$ induced from an immersed submanifold $\varphi(V)$ of $S^{n}$ of codimension $m+1>1$. Write the points of the unit normal bundle $B^{n-1}$ in the form $(v, \xi)$. As before, we consider the point sphere and great sphere maps,

$$
k_{1}(v, \xi)=(1, \varphi(v), 0), \quad k_{2}(v, \xi)=(0, \xi, 1) .
$$

To find the curvature spheres of $\lambda$, we use the following local trivialization of $B^{n-1}$ in a neighborhood of a given point $(v, \xi)$. Let $\xi_{0}, \cdots, \xi_{m}$ be orthonormal 
normal vector fields to $\varphi(V)$ on a normal coordinate neighborhood $W$ of $v$ which are parallel with respect to the normal connection along geodesics through $v$ in $W$. Arrange that $\xi_{0}(v)=\xi$. For any point $w \in W$ and unit normal $\eta$ to $\varphi(V)$ at $w$, we can write

$$
\eta=\left(1-\sum_{i=1}^{m} t_{\imath}^{2}\right)^{1 / 2} \xi_{0}+t_{1} \xi_{1}+\cdots+t_{m} \xi_{m},
$$

where $0 \leqq\left|t_{\imath}\right| \leqq 1$ for all $i$, and $t_{1}^{2}+\cdots+t_{m}^{2} \leqq 1$. The tangent space to $B^{n-1}$ at the given point $(v, \xi)$ can be considered to be

$$
T_{v} V \times \operatorname{Span}\left\{\frac{\partial}{\partial t_{1}}, \cdots, \frac{\partial}{\partial t_{m}}\right\}=T_{v} V \times \boldsymbol{R}^{m} .
$$

First note that $d k_{1}(0, Y)$ equals zero for any $Y \in \boldsymbol{R}^{m}$, since $k_{1}$ depends only on $v$. Hence, $k_{1}$ is a curvature sphere, as expected. Furthermore, since

$$
d k_{1}(X, 0)=(0, d \varphi(X), 0)
$$

is never in Span $\left\{k_{1}, k_{2}\right\}$ for a non-zero $X$ in $T_{v} V$, the multiplicity of the curvature sphere $k_{1}$ is $m$. If we let $K_{g}=\mu_{g} k_{1}+k_{2}$ be this curvature sphere, then we must take $\mu_{g}=\infty$ to get $k_{1}$. We find the other curvature spheres at $(v, \xi)$ by computing

$$
d\left(\mu k_{1}+k_{2}\right)(X, 0)=(0, \mu d \varphi(X)+d \xi(X), 0),
$$

where $\xi$ has been extended to a field of unit normals which is parallel with respect to the normal connection along geodesics through $v$. Thus, we see that $\mu k_{1}+k_{2}$ is a curvature sphere with principal vector $(X, 0)$ if and only if $\mu$ is a principal curvature of the shape operator $A_{\xi}$ with principal vector $X$. We summarize these results in the following lemma.

LEMMA 2. Let $\lambda: B^{n-1} \rightarrow \wedge^{2 n-1}$ be the Legendre submanifold induced from an immersed submanifold $\varphi(V)$ in $S^{n}$ of codimension $m+1>1$. Let $k_{1}$ and $k_{2}$ be the point sphere and great sphere maps for $\lambda$ given by (2.4). Then the curvature spheres of $\lambda$ at a point $(v, \xi)$ in $B^{n-1}$ are

$$
K_{\imath}=\mu_{\imath} k_{1}+k_{2}, \quad 1 \leqq \imath \leqq g,
$$

where $\mu_{1}, \cdots, \mu_{g-1}$ are the distinct principal curvatures of the shape operator $A_{\hat{\xi}}$ and $\mu_{g}=\infty$. For $1 \leqq i \leqq g-1$, the multrplicity of $K_{2}$ equals the multiplicity of $\mu_{\imath}$, while the multiplicity of $K_{g}$ is $\mathrm{m}$.

Now consider the case where $\varphi(V)$ is an immersed submanifold of $S^{n}$ of codimension $m+1$ and $U$ is a connected open subset of the unit normal bundle $B^{n-1}$ on which there are three distinct principal curvatures $\mu_{1}<\mu_{2}<\mu_{3}$ at each point. By Lemma 2, the induced Legendre submanifold $\lambda: B^{n-1} \rightarrow \wedge^{2 n-1}$ has four distinct curvature spheres at each point of $U$, and the Lie curvature $\Psi$ of 
$\lambda$ on $U$ is given by

$$
\Psi=\frac{\left(\mu_{4}-\mu_{3}\right)\left(\mu_{1}-\mu_{2}\right)}{\left(\mu_{4}-\mu_{2}\right)\left(\mu_{1}-\mu_{3}\right)}=\frac{\mu_{1}-\mu_{2}}{\mu_{1}-\mu_{3}}=\Phi,
$$

since $\mu_{4}=\infty$. The function $\Phi$ was called the Moebius curvature of the submanifold $\varphi$ by Pinkall and Thorbergsson [14]. The calculations of Miyaoka [8] show that $\Phi$ is invariant under Moebius transformations, i.e., Lie sphere transformations which take point spheres to point spheres.

Remark 3. For sufficiently small $t$, a tube $\varphi_{t}: B^{n-1} \rightarrow S^{n}$ of radius $t$ over $\varphi$ is an immersed hypersurface having $\varphi(V)$ as one of its focal submanifolds. One can compute the principal curvatures of $\varphi_{t}$ from those of $\varphi$ (see [5, pp. 131-132]) and then compute the Lie curvature $\Psi$ of $\varphi_{t}$. However, since the Legendre submanifolds induced by $\varphi_{t}$ and $\varphi$ are Lie equivalent, they have the same Lie curvatures. It is often easier to compute the Lie curvature of the hypersurface $\varphi_{t}$ by computing the Lie curvature of the Legendre submanifold induced by its focal submanifold $\varphi(V)$ using (2.5).

For example, consider the case of an isoparametric hypersurface $M$ in $S^{n}$ with four distinct principal curvatures at each point. $M$ is a tube of constant radius over each of its focal submanifolds in $S^{n}$. If $\varphi(V)$ is one of these focal submanifolds, then the results of Münzner [11] show that for each unit normal $\xi$ to $\varphi(V)$ at each point $\varphi(v)$, the shape operator $A_{\xi}$ has three distinct principal curvatures, $\mu_{1}=-1, \mu_{2}=0, \mu_{3}=1$. By Lemma 2 and formula (2.5), the Lie curvature $\Psi$ on $M$ has the constant value $1 / 2$. In terms of projective geometry, this means that for each $x \in M$, the four curvature spheres are a harmonic set on a projective line on the quadric $Q^{n+1}$.

Examples with $\Psi=1 / 2$.

Let $\varphi: V \rightarrow S^{n-m}$ be a Dupin hypersurface with field of unit normals $\xi$ such that $\varphi$ has three distinct principal curvatures $\mu_{1}<\mu_{2}<\mu_{3}$ at each point of $V$. Embed $S^{n-m}$ as a totally geodesic submanifold in $S^{n}$, and let $B^{n-1}$ be the unit normal bundle of the submanifold $\varphi(V)$ of codimension $m+1$ in $S^{n}$. Let $\lambda: B^{n-1}$ $\rightarrow \wedge^{2 n-1}$ be the Legendre submanifold induced from the submanifold $\varphi(V)$ in $S^{n}$. Any unit normal $\eta$ to $\varphi(V)$ in $S^{n}$ at a point $v \in V$ can be written as

$$
\eta=\cos \theta \xi(v)+\sin \theta N,
$$

where $N$ is a unit normal to $S^{n-m}$ in $S^{n}$. Then the shape operator

$$
A_{\eta}=\cos \theta A_{\xi}+\sin \theta A_{N}=\cos \theta A_{\xi},
$$

since $A_{N}=0$. Thus, the principal curvatures of $A_{\eta}$ are

$$
\alpha_{\imath}=\cos \theta \mu_{\imath}, \quad 1 \leqq i \leqq 3 .
$$


We see that if $\eta \cdot \xi=\cos \theta \neq 0$, then $A_{\eta}$ has three distinct principal curvatures, whereas if $\eta \cdot \xi=0$, then $A_{\eta}$ has only one distinct principal curvature 0 . Let $U$ be the open subset of $B^{n-1}$ on which $\cos \theta>0$, and let $\lambda_{U}$ denote the restriction of $\lambda$ to $U$. By Lemma 2, the Legendre submanifold $\lambda_{U}$ has four distinct curvature spheres at each point of $U$. Since $\varphi(V)$ is Dupin in $S^{n-m}$, it is easy to show that $\lambda_{U}$ is also Dupin (see the tube construction in [1] for more detail). Furthermore, by equation (2.5), the Lie curvature of $\lambda_{U}$ at a point $(v, \eta)$ of $U$ as in (2.6) is given by

$$
\Psi(v, \eta)=\Phi(v, \eta)=\frac{\alpha_{1}-\alpha_{2}}{\alpha_{1}-\alpha_{3}}=\frac{\cos \theta \mu_{1}-\cos \theta \mu_{2}}{\cos \theta \mu_{1}-\cos \theta \mu_{3}}=\frac{\mu_{1}-\mu_{2}}{\mu_{1}-\mu_{3}} .
$$

Now suppose that $\varphi(V)$ is a minimal isoparametric hypersurface in $S^{n-m}$ with three distinct constant principal curvatures. By Münzner [11], these principal curvatures must have the values $\mu_{1}=-\sqrt{3}, \mu_{2}=0, \mu_{3}=\sqrt{3}$. On the open subset $U$ of $B^{n-1}$ described above, the Lie curvature of the induced Dupin submanifold $\lambda_{U}$ has the constant value $1 / 2$ by (2.7). To construct an immersed proper Dupin hypersurface in $S^{n}$ with $g=4$ and $\Psi=1 / 2$, we simply take the open subset $\varphi_{t}(U)$ of the tube of radius $t$ around $\varphi(V)$ in $S^{n}$.

These examples are not Lie equivalent to an open subset of an isoparametric hypersurface with four principal curvatures in $S^{n}$ because they are reducible in the sense of Pinkall [12, p. 437] to the lower dimensional Dupin hypersurface $\varphi(V)$ in $S^{n-m}$, whereas an isoparametric hypersurface with $g=4$ is not reducible [1]. This fact is a consequence of the following simple geometric argument. Note that the curvature sphere $k_{1}$ of $\lambda$ lies in the linear subspace of codimension $m+1$ in $P^{n+2}$ orthogonal to the space spanned by $e_{n+3}$ and those vectors $N$ normal to $S^{n-m}$ in $S^{n}$. This implies that there are only two distinct curvature spheres on the lines $\lambda(v, N)$ for such $N$. On the other hand, if $\gamma: M^{n-1} \rightarrow \wedge^{2 n-1}$ is the Legendre submanifold induced by an isoparametric hypersurface with $g=4$ in $S^{n}$, then there are four distinct curvature spheres on each line $\gamma(x)$ for $x \in$ $M^{n-1}$. Thus no curvature sphere of $\gamma$ lies in the orthogonal complement in $P^{n+2}$ of an $(m+1)$-plane with signature $(1, m)$, and $\gamma$ cannot be Lie equivalent to $\lambda_{U}$. Of course, this change in the number of distinct curvature spheres of $\lambda$ at points of the form $(v, N)$ is precisely why the example $\lambda_{U}$ cannot be completed to a compact proper Dupin submanifold with $g=4$.

With regard to Theorem 1, we see that the example $\lambda_{U}$ comes as close as possible to satisfying the requirements for being Lie equivalent to an isoparametric hypersurface without actually fulfilling them. Specifically, the point sphere map $k_{1}=K_{4}$ corresponding to $\mu_{4}=\infty$ and the great sphere map $k_{2}=K_{2}$ corresponding to $\mu_{2}=0$ are both curvature spheres of $\lambda_{U}$. We have $\left\langle k_{1}, e_{n+3}\right\rangle=0$ and $\left\langle k_{2}, e_{1}\right\rangle=0$. If a third curvature sphere, say $K_{1}$, were to satisfy $\left\langle K_{1}, P_{1}\right\rangle$ $=0$ for a point $P_{1}$ on the timelike line $\left[e_{1}, e_{n+3}\right]$, then the corresponding principal curvature $\mu_{1}$ would be constant on $U$. This and the fact that $\Phi=1 / 2$ on $U$ would imply that $\mu_{3}$ is also constant on $U$ and that $\lambda_{U}$ is Lie equivalent to an open subset of an isoparametric hypersurface. 
Using this same method, it is easy to construct a proper Dupin hypersurface with $g=4$ and $\Psi=c$ for any number $0<c<1$. Note that formula (2.3) and the ordering of the principal curvatures imply that $0<\Psi<1$ always. If $\varphi(V)$ is an isoparametric hypersurface in $S^{n-m}$ with three distinct principal curvatures, then by Münzner [11], these must have the values

$$
\mu_{1}=\cot (\theta+(2 \pi / 3)), \quad \mu_{2}=\cot (\theta+(\pi / 3)), \quad \mu_{3}=\cot \theta, \quad 0<\theta<\pi / 3,
$$

and any value of $\theta$ in $(0, \pi / 3)$ can be realized by some hypersurface in a parallel family of isoparametric hypersurfaces. A direct calculation then shows that the Moebius curvature

$$
\Phi=\frac{\mu_{1}-\mu_{2}}{\mu_{1}-\mu_{3}}=\frac{1}{2}+\frac{\sqrt{3}}{2} \tan (\theta-(\pi / 6))
$$

on $\varphi(V)$, and this can assume any real value $c$ in $(0,1)$ by an appropriate choice of $\theta$ in $(0, \pi / 3)$. An open subset of a tube over $\varphi(V)$ in $S^{n}$ is a proper Dupin hypersurface with $g=4$ and $\Phi=c$.

Finally, note that (2.8) shows that two hypersurfaces in a parallel family of isoparametric hypersurfaces are not Moebius equivalent. Of course, this can easily be deduced directly from the fact that parallel transformation is not a Moebius transformation.

\section{REFERENCES}

[1] T. Cecil, Reducible Dupin submanifolds, to appear in Geom. Dedicata.

[2] T. CECIL, AND S.-S. CHERN, Tautness and Lie sphere geometry, Math. Ann. 278 (1987), 381-399.

[3] T. CECIL, AND S.-S. CHERN, Dupin submanifolds in Lie sphere geometry, Differential Geometry and Topology, Proceedings Tianjin 1986-87, Lecture Notes in Math 1369, 1-48, Springer, Berlin, 1989.

[4] T. Cecil, AND P. Ryan, Focal sets, taut embeddings and the cyclides of Dupin, Math. Ann. 236 (1978), 177-190.

[5] T. CEcil AND P. Ryan, Tight and taut immersions of manifolds, Research Notes in Mathematics 107, Pitman, London, 1985.

[6] K. Grove And S. Halperin, Dupin hypersurfaces, group actions and the double mapping cylinder, J. Differential Geometry 26 (1987), 429-459.

[7] R. MiуаокA, Compact Dupin hypersurfaces with three principal curvatures, Math. Z. 187 (1984), 433-452.

[8] R. MryaokA, Dupin hypersurfaces and a Lie invariant, Kodai Math. J. 12 (1989), 228-256.

[9] R. MryaokA, Dupin hypersurfaces with six principal curvatures, to appear in Kodai Math. J.

[10] R. Mryaoka And T. Ozawa, Construction of taut embeddings and Cecil-Ryan conjecture, Geometry of manifolds (edited by K. Shiohama), Acad. Press (1989), 181-189.

[11] H.F. MunZneR, Isoparametrische Hyperflächen in Sphären I and II, Math. Ann. 251 (1980), 57-71 and 256 (1981), 215-232. 
[12] U. Pinkall, Dupin hypersurfaces, Math. Ann. 270 (1985), 427-440.

[13] U. Pinkall, Dupinsche Hyperflächen in $E^{4}$, Manuscripta Math. 51 (1985), 89-119.

[14] U. Pinkall and G. Thorbergsson, Deformations of Dupin hypersurfaces, 1988 preprint, Technische Universität Berlin and University of Notre Dame.

[15] G. Thorbergsson, Dupin hypersurfaces, Bull. London Math. Soc. 15 (1983), 493498.

Thomas E. Cecil

Department of Mathematics

College of the Holy Cross

WORCESTER, MA 01610 U.S.A. 
